\title{
Low Vacuum Scanning Electron Microscopy: A Review of Progress \& Applications
}

\author{
D.J. Stokes
}

Polymers \& Colloids Group, University of Cambridge, Department of Physics, Cavendish Laboratory, Madingley Road, Cambridge, CB3 0HE, UK

Low vacuum imaging can be applied to a broad range of insulating specimens. Dry insulators include polymers, hard tissues such as wood \& bone, rocks, porous materials, minerals \& gemstones, ceramics and dry foodstuffs, for example. 'Dry' can also include specimens in which water is tightly bound or frozen, or where specimens are protected by a hard or waxy exterior. If water vapor is used as the imaging gas, in conjunction with thermal control, hydrated specimens such as cells \& tissues and heterogeneous liquids \& soft solids can be studied. An appealing feature of low vacuum instruments is their high throughput capability, particularly for dry specimens, facilitated by the elimination of preparatory steps. The largely non-destructive nature of low vacuum SEM means that specimens can be either re-used for further experiments or studied using complementary techniques such as TEM, AFM and confocal microscopy. The absence of a coating means that the native surface can be explored, leading to such possibilities as dynamic experiments and exploitation of unique contrast phenomena.

Specimen-dependent SE contrast, particularly in the case of low-Z specimens, can aid the identification of different phases within heterogeneous materials [1]. However, it has been noted that the interpretation of signals emanating from dielectric materials is not always straightforward: a number of factors can influence emission and detection. For example, localized charging can lead to transient effects on signal intensity [2-4], the detector field strength can be altered by both positive ions and the dielectric properties of the specimen, and positive ions can scavenge signals by recombining with low energy SE emitted by the specimen or becoming 'pinned' to the surface [5-7]. Used properly, these phenomena can make a significant contribution to the characterisation of a diverse range of materials.

General applications of low vacuum SEM are many \& varied, and there is still plenty of potential for development, in tandem with emerging technologies such as tissue engineering, bio-nanotechnology and organic optoelectronics. For example, low vacuum methodologies are playing an active part in elucidating the mechanisms underlying physiological responses to biomimetic materials for bone tissue engineering [8]. We can study in vitro cellular morphology, attachment and proliferation on biomaterial substrates and observe bone-implant interfaces to quantify bone ingrowth around implanted materials following in vivo experiments, in conjunction with x-ray microanalysis. Figure 1 shows the early development of a physiological apatite layer, formed by immersion of a bioceramic in simulated body fluid.

There is also scope for new methodologies for dynamic observations. Recent work has focused on low temperature experiments, where uncoated frozen specimens can be made to undergo controlled sublimation in low vacuum, and very small partial pressures of nitrogen gas can be used to obtain high-quality images down to cryogenic temperatures [9]. The use of a mixed gas environment (e.g. water vapor and nitrogen or nitrous oxide) is also under development, with the aim of combining 
thermodynamic control and imaging capability, for studying a range of sub-zero processes in situ. These might include structure evolution during freeze-drying, ice crystal nucleation, growth and morphology, controlled etching and sublimation, freeze-thaw or thermal cycling and phase transitions. Applications could include foods, pharmaceutical products, cosmetics, cement and stone, and biological, environmental and atmospheric systems such as frost-forming bacteria, the distribution of ice-adsorbed impurities in clouds and the conditions determining the shapes of snowflakes. Figure 2 shows the vapor deposition of ice crystals in a nitrogen atmosphere at a temperature of $-65^{\circ} \mathrm{C}$.

\section{References}

[1] D.J. Stokes et al., Langmuir, 14(16) (1998) 4402-4408.

[2] B.J. Griffin, Microsc. Microanal. 3 (Suppl. 2) (1997) 1197-1198.

[3] E. Doehne, Microsc. Microanal. 4 (Suppl 2) (1998) 292-293.

[4] D.J.Stokes et al., Scanning, 22(6) (2000) 357-365.

[5] J.P. Craven et al., J. Microscopy, 205(1) (2002) 96-105.

[6] M. Toth et al., J. Microscopy, 205(1) (2002) 86-95.

[7] M. Toth, et al., J. Applied Physics, 2002, 91(7), p.4479-4491 and p.4492-4499.

[8] D.J. Stokes et al., Mat. Res. Soc. Symp. Proc. (711) (2002) 113-118.

[9] D.J. Stokes et al., J. Microscopy, (2003) (submitted).

[10] This work was funded by a Royal Society Warren Research Fund Dorothy Hodgkin Research

Fellowship. The loan of a Gatan Alto 2500 Cryo-transfer system is gratefully acknowledged.

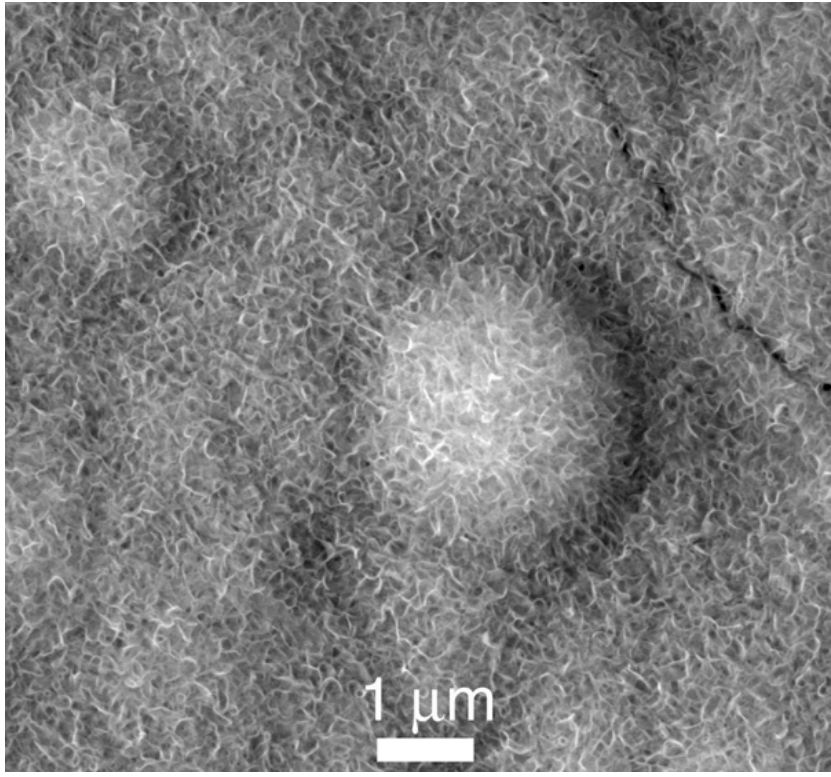

Figure 1. Low vacuum imaging of apatite formation on a bioceramic substrate. Fine microstructures are visible (dimensions on the order of 200nm x $10 \mathrm{~nm}$. Magnification $\mathrm{x} 10,000)$. Specimen courtesy of Claudia Bothelo.

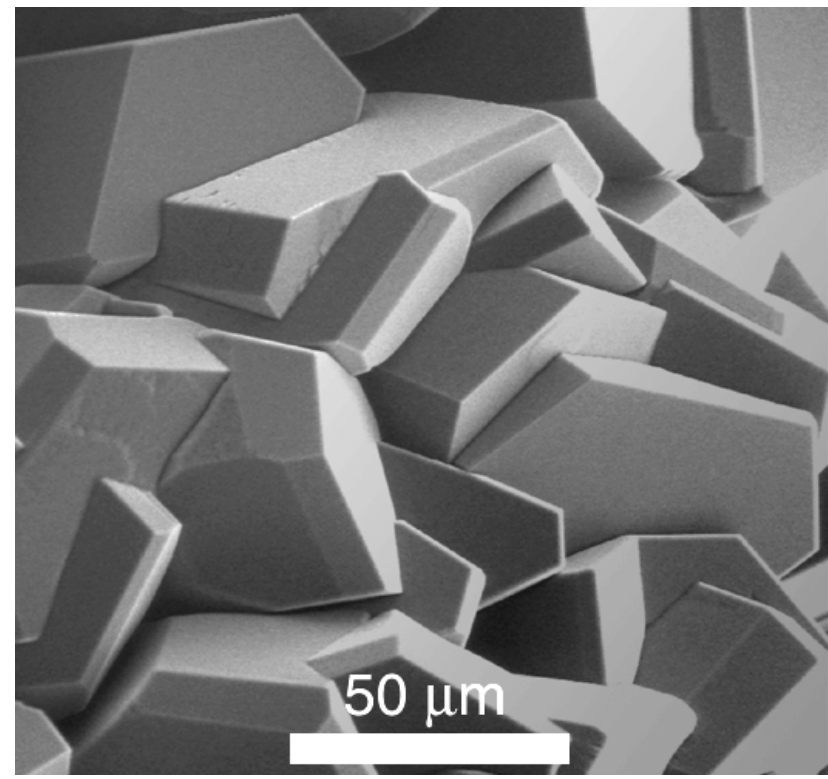

Figure 2. In situ vapor deposition of ice: a small partial pressure of nitrogen is mixed with a similar amount of water vapor, with $\mathrm{T}$ lowered to $-65^{\circ} \mathrm{C}$. Water vapor precipitates out of the gas phase, while nitrogen serves as the imaging gas. 